\title{
Lattice and magnetic dynamics in the polar, chiral, and incommensurate antiferromagnet $\mathrm{Ni}_{2} \mathrm{InSbO}_{6}$
}

\author{
M. A. Prosnikov $\odot,{ }^{1, *}$ A. N. Smirnov $\odot,{ }^{1}$ V. Yu. Davydov, ${ }^{1}$ Y. Araki $\odot,{ }^{2}$ T. Arima,${ }^{2}$ and R. V. Pisarev ${ }^{1}$ \\ ${ }^{1}$ Ioffe Institute, Russian Academy of Sciences, 194021 St.-Petersburg, Russia \\ ${ }^{2}$ Department of Advanced Materials Science, University of Tokyo, Japan
}

(Received 1 July 2019; revised manuscript received 15 September 2019; published 10 October 2019)

\begin{abstract}
Complex systems with coexisting polarity, chirality, and incommensurate magnetism are of great interest because they open new degrees of freedom in the interaction between crystal and magnetic subsystems and therefore they host a plethora of intriguing physical properties. Here we report the optical properties and lattice and spin dynamics of $\mathrm{Ni}_{2} \mathrm{InSbO}_{6}$ single crystals. This material was studied with the use of polarized optical

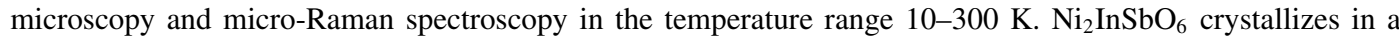
polar structure described by the noncentrosymmetric space group $R 3$ and the two types of structural domains were visualized due to the natural optical activity of opposite chirality. Raman tensor elements of most $A$ and $E$ phonons along with their symmetry were determined. The manifestation of LO-TO splitting was observed for the $A$ modes. By tracking the temperature dependencies of phonon frequencies, the well pronounced spin-phonon interaction was observed for several modes below and above the Néel transition temperature $T_{N}=76 \mathrm{~K}$. In the antiferromagnetic phase, a broad excitation centered at $247 \mathrm{~cm}^{-1}$, was detected and assigned to the two-magnon mode and this value was used for estimating exchange parameters through linear spin-wave theory calculations.
\end{abstract}

DOI: $10.1103 /$ PhysRevB.100.144417

\section{INTRODUCTION}

Breaking of the space inversion symmetry in crystals gives rise to various intriguing phenomena. For example, it allows the linear electro-optic effect, the piezoelectric effect, the optical second harmonic generation of the electric dipole type, etc. [1-3]. In particular, the macroscopic electric polarization becomes allowed which strongly affects the lattice dynamics, e.g., by inducing the giant LO-TO splitting in ferroelectrics [4], or leading to the presence of oblique phonon modes for which the frequency continuously varies as a function of phonon propagation vector (angular dispersion) [5]. Another interesting property of some noncentrosymmetric crystals is the chirality, which is usually manifested as the natural optical activity in uniaxial crystals, and directional effects, such as circular or linear dichroism, also called optical diode effects [6-8]. Moreover, in magnetically ordered systems the timereversal symmetry is broken which results in new degrees of freedom for manifestation and control of their physical properties with external magnetic and electric fields or by varying the temperature in the vicinity of phase transitions.

Antiferromagnets with complex crystal and magnetic structures are promising candidates in the emerging fields of antiferromagnetic spintronics [9-12] and sub-terahertz and terahertz magnonics [13] due to a usually richer excitation spectrum, much higher resonance frequencies, and the absence of stray field in comparison to ferromagnets and ferrimagnets. In multiferroic and magnetoelectric crystals and artificial structures both space and time-reversal symmetries

\footnotetext{
*Present address: High Field Magnet Laboratory (HFML-EMFL), Radboud University, Toernooiveld 7, 6525 ED Nijmegen, The Netherlands; mikhail.prosnikov@ru.nl.
}

are simultaneously broken pushing further the interest to magnetically ordered systems due to a coupling of multiple order parameters [14-20].

$\mathrm{Ni}_{2} \mathrm{InSbO}_{6}$ crystals have a nonecentrosymmetric structure and therefore combine several of the above mentioned properties, namely the polarity, chirality, and incommensurate antiferromagnetic structure [21]. All these properties make this compound a unique playground for observation of a plethora of effects, elementary excitations, and interactions between them. $\mathrm{Ni}_{2} \mathrm{InSbO}_{6}$ belongs to a group of trigonal corundumlike crystals with general formula $\mathrm{A}_{2} \mathrm{BB}^{\prime} \mathrm{O}_{6}$ [22]. We note that this structural type is radically different from the various crystal structures realized in the so-called double perovskites for which the similar abbreviation is used [23]. Up to now, mostly structural and static magnetic properties of the corundum-type magnetic compounds were studied and the most explored system is $\mathrm{Ni}_{3} \mathrm{TeO}_{6}[24,25]$ which possesses a complex magnetic phase diagram [26]. From the point of view of magnetic properties, the important difference between $\mathrm{Ni}_{3} \mathrm{TeO}_{6}$ and $\mathrm{Ni}_{2} \mathrm{InSbO}_{6}$ is that in the former the magnetic structure is commensurate [27] while in the latter it is incommensurate [21].

Raman scattering is a well established and powerful technique allowing one to probe simultaneously the lattice, electronic, magnetic, superconducting, and other types of excitations as well as the coupling between them. Additionally, polarization dependent experiments allow one to directly determine the symmetry of excitations and analyze Raman tensor elements. It is important to remind that the mutual exclusion principle is not applicable for noncentrosymmetric systems and therefore Raman scattering allows probing of odd (polar) and even (unpolar) phonons in a single experiment. There is a growing interest in the phase difference formalism 
of Raman tensor elements in optically anisotropic media supported by discussion of the origin and intrinsicality of such phases [28-30]. We note that most of the experimental observations are limited to two-dimensional materials [31,32] and bulk crystals with relatively simple structures [33,34].

In this paper we report on a comprehensive experimental study of the optical properties, lattice, and magnetic dynamics of high-quality $\mathrm{Ni}_{2} \mathrm{InSbO}_{6}$ single crystals within paramagnetic and antiferromagnetic phases. The frequencies of polar lattice modes are the basic ingredients for the quantitative investigation of dielectric properties of the system. Detailed phonon spectra may serve as a consistent experimental reference and starting point for computations on $\mathrm{Ni}_{2} \mathrm{InSbO}_{6}$ and related crystals based on density functional theory (DFT) [35]. We performed Raman scattering measurements that allowed us to determine the relevant tensor elements and complex phases for the $A$-symmetry phonons. The study of magnetic excitations is essential for understanding of the ground and excited states of the spin system and, consequently, for deriving microscopic parameters, such as exchange couplings and single-ion anisotropy (SIA). Experimental observation of a two-magnon mode was interpreted with the use of linear spin-wave theory calculations.

The paper is organized as follows. Section II describes the single crystals growth process and other experimental details. In Sec. III results and their interpretation are presented, starting from the absorption spectroscopy measurements in the region of $d-d$ transitions in $\mathrm{Ni}^{2+}$ ions. Section III $\mathrm{B}$ is devoted to the optical activity study observed as a rotation of the linearly polarized light traversing the sample. These results are followed by those of Raman scattering on lattice excitations and manifestations of the spin-phonon interaction. Section III ends with results on magnetic dynamics followed by the interpretation within the linear spin-wave theory approximation. Section IV summarizes experimental and theoretical results and ends with conclusions.

\section{EXPERIMENTAL DETAILS}

Single crystals were grown by the chemical vapor transport method. The powder samples of $\mathrm{Ni}_{2} \mathrm{InSbO}_{6}$ were synthesized by solid-phase reaction technique and put into an evacuated tube with $\mathrm{PtCl}_{2}$ as a transport agent [36]. The tube was placed for two weeks into a horizontal furnace with temperatures of 1080 and $1000{ }^{\circ} \mathrm{C}$ for source and growth sides of the tube, respectively. Grown crystals were typically of a $1 \times$ $1 \times 0.15 \mathrm{~mm}^{3}$ size. They are characterized by a deep-green color specific for many crystals with $\mathrm{Ni}^{2+}$ ions in oxygen $\mathrm{O}^{2-}$ octahedral coordination [37-41]. The following notation of coordinate systems is used below: the $x, y$, and $z$ axes coincide with the $a, b$, and $c$ crystallographic axes in the hexagonal setting, while the $X, Y$, and $Z$ axes are used in the orthogonal setting, where $X$ and $Z$ coincide with $x$ and $z$, and $Y$ is perpendicular to the $(x z)$ plane.

Polarized optical microscopy and conoscopy measurements were done at room temperature with the use of a BX53 (Olympus) microscope. Room temperature unpolarized optical absorption spectra were recorded with the use of the UV-3600 Plus (Shimadzu) spectrometer equipped with a $0.8 \mathrm{~mm}$ diaphragm.
Angular-resolved azimuthal Raman spectra were measured with the use of a T64000 (HORIBA Jobin-Yvon) spectrometer equipped with a liquid-nitrogen-cooled CCD camera. The 532 $\mathrm{nm}(2.33 \mathrm{eV})$ line of a Torus Nd:YAG-laser (Laser Quantum) was used as the excitation source. A 50x objective was employed for focusing the incident beam and collecting the scattered light. Temperature-dependent spectra were recorded using a closed-cycle helium cryostat (Cryo Industries) with temperature stability better than $1 \mathrm{~K}$. Samples were mounted with the use of silver paste for achieving good thermal contact. Calibration of the spectrometer was done with the $520.7 \mathrm{~cm}^{-1}$ phonon line of a Si single crystal. Measurements were done in the backscattering geometry for all informative polarization settings labeled according to the Porto notation rule, where $X(Y Z) \bar{X}$ stands for the case of incident light linearly polarized along the $Y$ axis, propagated and backscattered along the same $X$ axis, while the $Z$ component of scattered light is measured. Angular azimuthal dependencies of the spectra were measured at room temperature by rotating the polished single crystal cuts of different orientations.

\section{RESULTS AND DISCUSSION}

\section{A. Optical spectroscopy}

Unpolarized absorption spectrum of $\mathrm{Ni}_{2} \mathrm{InSbO}_{6}$ measured for the light propagating along the optical axis is shown in Fig. 1. Accuracy of the measurements in spectral regions of high optical absorption (saturation) was restricted by the thickness and small size of available samples. For determining the positions of the absorption bands, a curve fitting procedure $\left(R^{2}=0.907\right)$ with a simple model of four Gaussian curves was applied. Characteristic electronic $d-d$ transitions from the ground ${ }^{3} A_{2}$ state to the excited states of $\mathrm{Ni}^{2+}$ ions with

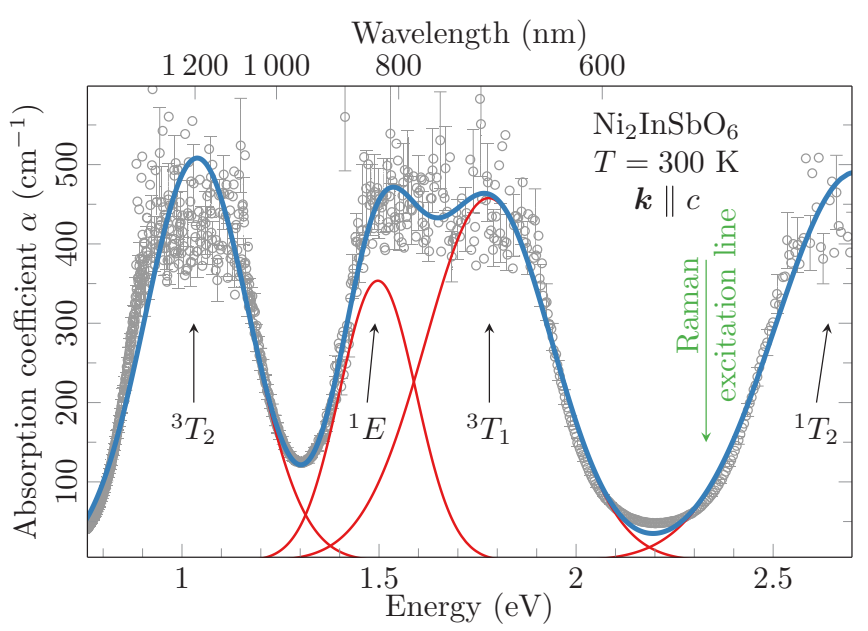

FIG. 1. Unpolarized room-temperature absorption spectrum of $\mathrm{Ni}_{2} \mathrm{InSbO}_{6}$ for the light propagating along the optical $Z$ axis. Observed absorption bands are due to electronic transitions within the $3 d^{8}$ states of $\mathrm{Ni}^{2+}$ ions split by the crystal field. Experimental data are shown by gray circles; modeling of the spectra was done using the sum of Gaussian curves (red) shown in blue. Black arrows mark individual transitions; the green arrow in the transparency region highlights the photon energy $2.33 \mathrm{eV}(532 \mathrm{~nm})$ of the excitation laser source used in the Raman experiments. 
energies $1.03,1.49,1.78$, and $2.67 \mathrm{eV}$ were observed. These values are noticeably smaller than $1.13,1.75,1.95$, and $2.69 \mathrm{eV}$ values observed in the textbook $\mathrm{NiO}$ crystal composed of only cubic $\left[\mathrm{NiO}_{6}\right]$ octahedra $[42,43]$. This observation of systematically lower positions of absorption bands is evidence of a weaker crystal field cubic parameter $D q$ in $\mathrm{Ni}_{2} \mathrm{InSbO}_{6}$ due to the larger Ni-O ionic distances in comparison to $\mathrm{NiO}$. Thus, the observed bands are assigned to ${ }^{3} \mathrm{~A}_{2} \rightarrow$ ${ }^{3} T_{2},{ }^{1} E,{ }^{3} T_{1},{ }^{1} T_{2}$ transitions. The absorption bands with similar energy positions and the same assignment were observed in a structurally related compound $\mathrm{Ni}_{3} \mathrm{TeO}_{6}$ [44]. The excitation source of $532 \mathrm{~nm}$ was chosen for Raman scattering experiments because the low absorption in the green transmittance region is favorable for maximizing the scattering volume and avoiding the resonant excitation conditions.

\section{B. Polarized microscopy}

Polarized optical microscopy is a powerful method for fast and robust determination of different optical parameters of single crystals. In our research, we applied this method for measuring the reciprocal effect of natural optical activity in the chiral $\mathrm{Ni}_{2} \mathrm{InSbO}_{6}$ single crystal. This effect is described by a third rank polar tensor $\gamma_{i j k}$ invariant under the timereversal symmetry operation which relates the light vector $\boldsymbol{k}$ with the dielectric impermittivity tensor $[1,45]$. The crystal structure of $\mathrm{Ni}_{2} \mathrm{InSbO}_{6}$ is described by the point group $3\left(\mathrm{C}_{3}\right)$ and therefore $\gamma_{i j k}=-\gamma_{j i k}$. For the linearly polarized light propagating along the optical axis $\boldsymbol{k}_{3} \| Z$, the natural optical activity is defined by two $\gamma_{123}=-\gamma_{213}$ tensor components and is manifested as a rotation of the polarization plane of the transmitted light.

Three images of a crystal sample in the green transparency region were taken with different angles between polarizer and analyzer as shown in Figs. 2(a)-2(c). The characteristic dark cross observed in conoscopic observations confirms that the sample is oriented with good accuracy along its optical axis. These images clearly show that the sample consists of several domains with opposite chirality because the polarization plane of transmitted light is rotated in opposite directions in different areas of the sample. Similar observations were reported for the $\mathrm{Ni}_{3} \mathrm{TeO}_{6}$ crystal which crystallizes in the same space group $R 3$ [46]. Figure 2(e) shows the intensity of transmitted light as a function of distance along the path marked in Fig. 2(c) for different angles between polarizer and analyzer. Dark regions of near-zero transmission for crossed polarizers $\left(90^{\circ}\right.$, brown curve) indicate compensation of optical rotation by two domains of opposite chirality placed one above another. Additionally, the presence of nonhorizontal regions in Fig. 2(e) suggests that some domain walls are not parallel to the optical $(Z)$ axis but tilted to it.

The observed sample images make it possible to determine the specific optical rotation angle of the sample with the known thickness by rotating the analyzer in order to find the transmission minimum. Such curves for selected regions are shown in Fig. 2(d). The specific rotation of the linearly polarized light transmitted through a crystal due to optical activity is given by $\alpha=\phi / l d$, where $\phi$ is the optical rotation angle, $l$ is the sample thickness, and $d$ is the density. For the largest rotation angle [red curve in Fig. 2(d)] we obtain
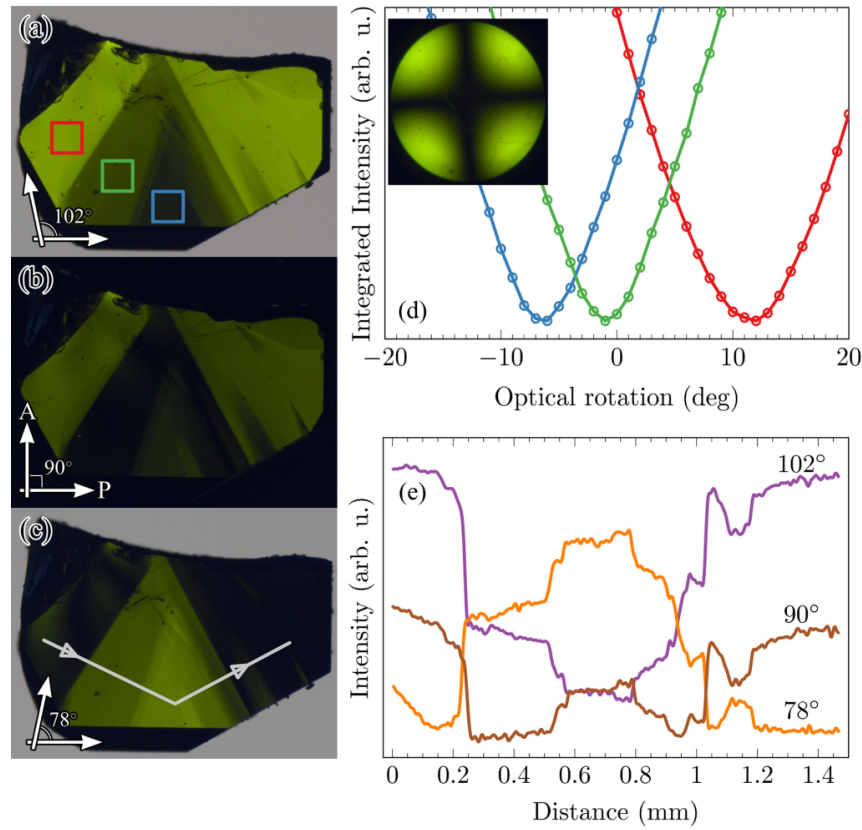

FIG. 2. (a)-(c) Polarized microscopy images of a $\mathrm{Ni}_{2} \mathrm{InSbO}_{6}$ sample obtained for the light propagating along the optical $Z$ axis and for different angles between the polarizer $(\mathrm{P})$ and the analyzer (A). (d) Integrated intensity of the regions marked in (a) as a function of the angle difference between the polarizer and the analyzer. Inset shows the conoscopic image of the sample along the optical axis. (e) Transmitted intensity profiles along the gray paths shown in (c) for different polarizer/analyzer angles.

$\alpha \approx 875^{\circ} \mathrm{dm}^{-1} \mathrm{~cm}^{3} \mathrm{~g}^{-1}$ which is lower than the value of $1355^{\circ} \mathrm{dm}^{-1} \mathrm{~cm}^{3} \mathrm{~g}^{-1}$ determined for $\mathrm{Ni}_{3} \mathrm{TeO}_{6}$ [46]. If these two notably different values are normalized to the number of $\mathrm{Ni}^{2+}$ ions per unit cell they become equal to each other with an accuracy better than $5 \%$. We note that a determined value of specific rotation is comparable with the $816^{\circ} \mathrm{dm}^{-1} \mathrm{~cm}^{3} \mathrm{~g}^{-1}$ value for the $\alpha$-quartz in the visible spectral range. Concluding this paragraph, it is interesting to note that though $\mathrm{Ni}_{2} \mathrm{InSbO}_{6}$ is a chiral crystal, it belongs to the achiral trigonal space group $R 3$ which was determined using the $\mathrm{x}$-ray diffraction $[21,36]$.

\section{Lattice dynamics}

Characterization of the lattice vibrational modes is essential for a better understanding of dielectric and polar properties of crystal. Lattice dynamics of $\mathrm{Ni}_{3} \mathrm{TeO}_{6}$ was previously characterized by infrared absorption measurements in [47], however no reports on LO-TO splitting of phonon modes in this crystal was reported. Additionally, the lattice dynamics of only $\left[\mathrm{TeO}_{6}\right]$ groups was studied by infrared reflectivity and Raman scattering in [48].

According to the group theoretical analysis, in the trigonal space group $R 3$ (No. $146, Z=3$ in a hexagonal cell) 18 modes are expected which are simultaneously active in the infrared (IR) and Raman processes according to Eq. (1):

$$
\Gamma=9 A+9 E .
$$


In this case the $A$ and $E$ modes of Raman tensors have the following forms:

$$
\begin{gathered}
A=\left(\begin{array}{ccc}
|a| e^{i \phi_{a}} & 0 & 0 \\
0 & |a| e^{i \phi_{a}} & 0 \\
0 & 0 & |b| e^{i \phi_{b}}
\end{array}\right) \\
E=\left(\begin{array}{ccc}
|d| e^{i \phi_{d}} & -|c| e^{i \phi_{c}} & -|f| e^{i \phi_{f}} \\
-|c| e^{i \phi_{c}} & -|d| e^{i \phi_{d}} & |g| e^{i \phi_{g}} \\
-|f| e^{i \phi_{f}} & |g| e^{i \phi_{g}} & 0
\end{array}\right) .
\end{gathered}
$$

The Raman scattering intensity is given by [49]

$$
I=\left|\boldsymbol{e}_{i} T \mathcal{R} T^{-1} \boldsymbol{e}_{s}\right|^{2},
$$

where $\boldsymbol{e}_{i}$ and $\boldsymbol{e}_{s}$ are the polarization vectors of the incident and scattered light, $T$ is the rotational matrix of the Euler angles, and $\mathcal{R}$ is the Raman tensor for the mode of particular symmetry given in Eqs. (2) and (3). In Raman scattering processes, the rotation of the polarization is equivalent to the rotation of the crystal, and the latter case was used in our experiments. The polarization vectors were fixed such as $\boldsymbol{e}_{i}$ equals $(1,0,0)$ or $(0,1,0)$ in the case of parallel or crossed polarizations, respectively, while $\boldsymbol{e}_{s}$ was fixed as $(1,0,0)$ in all experiments.

However, the presence of a finite electric polarization along the $c$ axis allowed by the absence of the inversion center results in the LO-TO splitting of the phonon modes followed by modification of the selection rules. Thus, the effective number of the observed modes should be greater than stated in Eq. (1). $A(\mathrm{LO})$ phonons should be observed only in parallel $Z(X X) \bar{Z} \leftrightarrow Z(Y Y) \bar{Z}$ type polarizations and characterized by a single Raman tensor element $b$ [see Eq. (2)], while the $A$ (TO) modes should be observed in any polarization for the light propagation direction perpendicular to the $Z$ axis [50]. It should be noted that the $A(\mathrm{LO})$ modes are inactive in the IR absorption and reflection processes, which makes Raman scattering advantageous for comprehensive characterization of the lattice dynamics. Analysis of the Raman tensor in optically anisotropic crystals requires taking into account the complex phases between the tensor elements, and usually phase differences are found to be close to $90^{\circ}$ [28].

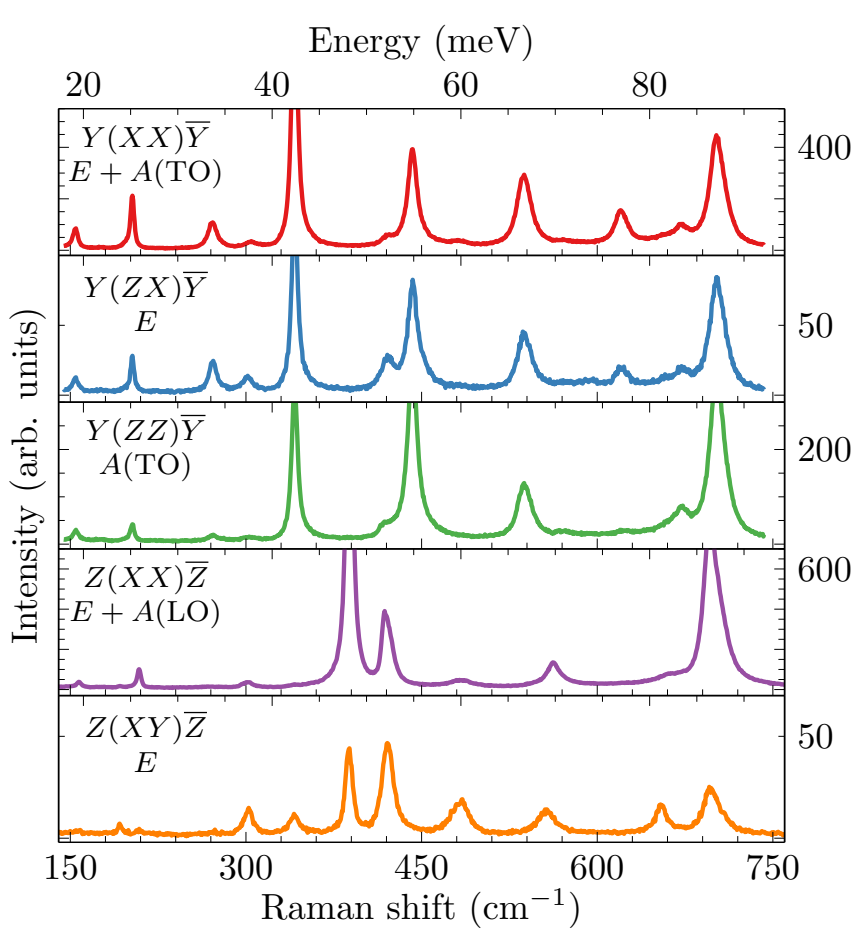

FIG. 3. Raman spectra measured for all required polarizations at room temperature. Note that the ordinate axis scales are different for each spectrum.

Raman spectra of $\mathrm{Ni}_{2} \mathrm{InSbO}_{6}$ lattice modes measured at room temperature are shown in Fig. 3. All observed phonon are lying in the $150-710 \mathrm{~cm}^{-1}$ spectral range. The presence of rather intense second-order phonon scattering is observed at frequencies above $1300 \mathrm{~cm}^{-1}$ additionally that indirectly confirms high structural quality of the single-crystal samples. All LO and TO phonon modes are active in the Raman scattering process and this circumstance complicates the analysis due to the strong overlapping between different modes. Both types of the $A$ and $E$ modes were observed, however the $E$ modes are more elusive, similar to those in the model trigonal ferroelectric $\mathrm{LiNbO}_{3}$ [51]. Because of a large number of nonzero $E$ Raman tensor elements and the weakness of such phonons, these elements and complex phases were determined only for

TABLE I. Frequency $\left(\mathrm{cm}^{-1}\right)$, FWHM $\left(\mathrm{cm}^{-1}\right)$, Raman tensor elements, phase difference $(\mathrm{deg})$, and spin-phonon coupling constant $\left(\mathrm{cm}^{-1}\right)$

\begin{tabular}{|c|c|c|c|c|c|c|c|c|c|c|c|c|}
\hline \multicolumn{10}{|c|}{$A(\mathrm{TO})[A(\mathrm{LO})]$} & \multicolumn{3}{|c|}{$E$} \\
\hline \multicolumn{2}{|c|}{$\omega$} & \multicolumn{2}{|c|}{ FWHM } & \multicolumn{2}{|c|}{$a$} & $\begin{array}{c}b \\
4.69\end{array}$ & $\begin{array}{l}\phi_{a-b} \\
87.7\end{array}$ & \multicolumn{2}{|c|}{$\lambda$} & $\begin{array}{c}\omega \\
192\end{array}$ & $\begin{array}{c}\text { FWHM } \\
5.6\end{array}$ & $\begin{array}{l}\lambda \\
\lambda \\
\end{array}$ \\
\hline 204 & [210] & 4.1 & [3.9] & 14.89 & [9.68] & 6.04 & 88.8 & - & - & - & - & - \\
\hline 342 & [389] & 6.2 & {$[6.6]$} & 33.74 & [43.24] & 19.01 & 88.8 & -0.55 & {$[-1.78]$} & 271 & 9.6 & - \\
\hline- & [419] & - & [5.5] & - & [15.08] & - & - & - & {$[-2.14]$} & $302^{\mathrm{a}}$ & - & - \\
\hline 621 & [662] & 11.9 & [20.7] & 11.76 & {$[4.21]$} & 2.80 & 80.2 & - & - & 484 & 14.3 & -0.68 \\
\hline 673 & [696] & 18.1 & [11.6] & 8.44 & [25.28] & 7.52 & 85.4 & - & - & - & - & - \\
\hline 702 & [706] & 12.4 & [17.9] & 16.65 & [16.98] & 15.88 & 91.1 & -1.13 & {$[-0.82]$} & - & - & - \\
\hline
\end{tabular}
for the observed phonon modes. Constants $\lambda$ are determined from temperature measurements, other parameters are given for the room temperature.

${ }^{\mathrm{a}}$ The values used to fit this mode are $c=3.8, d=3.7, e=3.8, f=3.4, \phi_{d}=45.8^{\circ}, \phi_{e}=-20.2^{\circ}, \phi_{e}=18.9^{\circ}, \phi_{f}=-51.9^{\circ}$. 

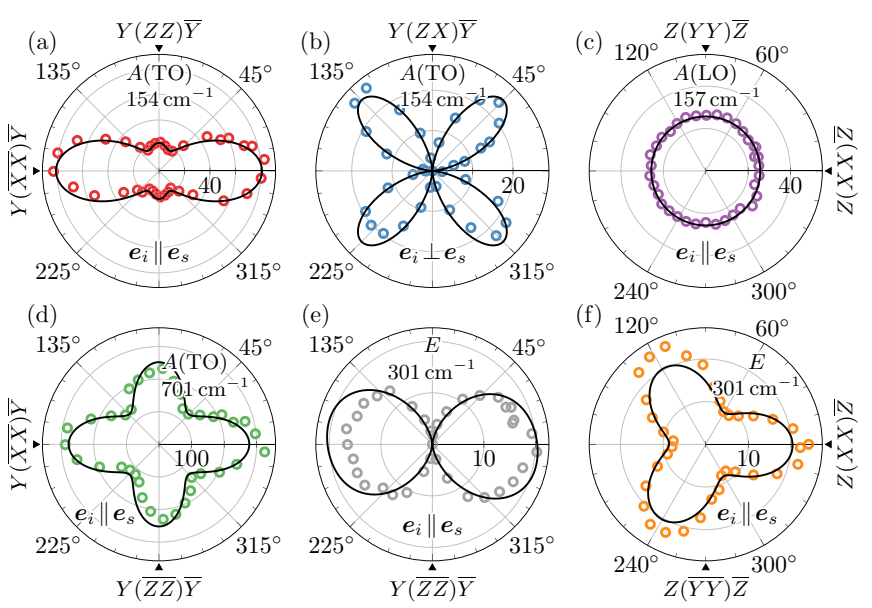

FIG. 4. Azimuthal dependencies of the intensity (arbitrary units) of selected phonons as a function of the polarization of the linearly polarized light. Colored circles represent experimental values, solid lines are fits calculated according to Eq. (4).

the $301 \mathrm{~cm}^{-1}$ mode as indicated in Table I. Due to similar reasons, the LO-TO splitting was reliably observed only for the $A$ modes. It should be noted that obtained frequency values of the LO-TO modes can be used for estimating the dielectric permittivities $\epsilon_{0}$ and $\epsilon_{\infty}$ using the Lyddane-Sachs-Teller relation [52] $\omega^{2}(\mathrm{LO}) / \omega^{2}(\mathrm{TO})=\epsilon_{0} / \epsilon_{\infty}$. The LO-TO frequency ratios for all the observed phonons are quite irregular [53], but this is expected for real anisotropic systems. Averaged value gives $\epsilon_{0} / \epsilon_{\infty}=1.10$.

To unambiguously determine the symmetry of phonons and extract Raman tensor elements we performed the measurements by varying the azimuthal angle of crystal with respect to linearly polarized incident light. Spectra were fitted with Voigt profiles and linear background and typically resulting values of $R^{2}$ were $\geqslant 0.98$. For each observed phonon the fitting was done simultaneously for all available polarizations, e.g., (a) and (b), or (e) and (f) plots in Fig. 4. This approach allowed us to extract individual tensor elements and phases. Necessity to use complex phases in the case of the $\mathrm{Ni}_{2} \mathrm{InSbO}_{6}$ crystal is directly confirmed by nonvanishing intensity of the $A$ (TO) modes when measured at $45^{\circ}$ to the $X$ (or $Y$ ) axis, as can be seen for the $154 \mathrm{~cm}^{-1}$ mode in Fig. 4. All complex phases for the $A$ modes were close to expected $90^{\circ}$ value. The deviations from this value were mainly observed in few- and single-monolayer thickness samples [32]. Estimated Raman tensor elements along with phase differences are summarized in Table I. We should note that the absolute values of tensor elements are instrument dependent, so for comparison with other experiments $a / b$ ratio should be used.

No frequency shifts of more than $2 \mathrm{~cm}^{-1}$ were observed in angular-resolved measurements which rules out the presence of oblique modes observed, e.g., in $\mathrm{BiFeO}_{3}$ [5]. We suppose that the absence or smallness of the frequency shifts could be due to a small value of the electric polarization $6 \mu \mathrm{C} / \mathrm{cm}^{2}$ of $\mathrm{Ni}_{2} \mathrm{InSbO}_{6}$ [36] in comparison with other polar systems where angular dispersion shifts were clearly observed $[54,55]$.

\section{Spin-phonon coupling}

Temperature dependent experiments revealed well pronounced frequency shifts of some phonon modes above and
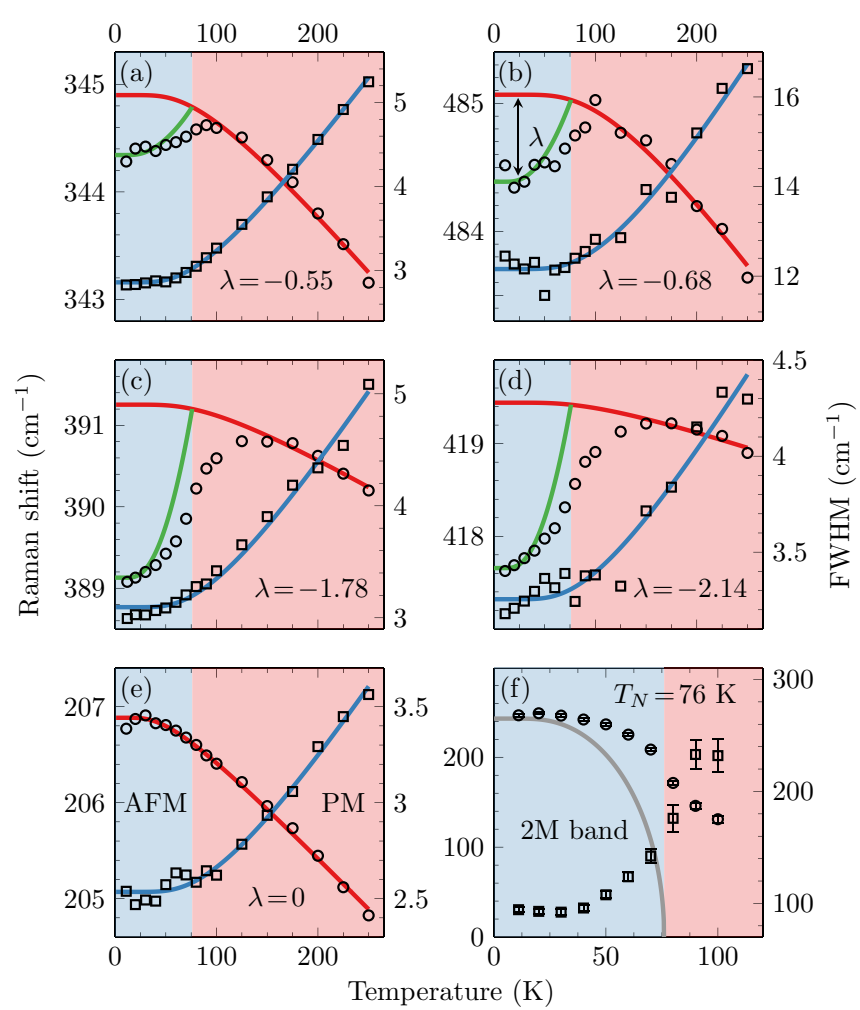

FIG. 5. Temperature dependencies of frequency (circles) and FWHM (squares) of (a)-(e) phonon and (f) two-magnon excitations. Blue and red regions correspond to antiferromagnetic and paramagnetic phases. Red and blue curves are anharmonic contributions according to Eqs. (5) and (6). Green curves are spin-phonon contribution. Gray curve is the Brillouin function for $S=1$.

below $T_{N}$. Temperature dependencies of the frequency and half-width of several particular phonons are shown in Fig. 5. Typically, the conventional behavior of phonon modes with cooling is characterized by simultaneous narrowing and hardening due to thermal suppression of anharmonism, see, e.g., Fig. 5(e). The anharmonic curves for both frequency and halfwidth in $\mathrm{Ni}_{2} \mathrm{InSbO}_{6}$ were calculated with the use of the well known equations [56]. For explaining the observed behavior, the three phonon relaxation process was found to be sufficient. This process is subject to the following theoretical predictions for the frequency and half-width:

$$
\begin{aligned}
& \omega_{i}(T)=\omega_{i 0}-A\left(1+\frac{2}{e^{\hbar \omega_{i 0} / 2 k_{B} T}-1}\right), \\
& \Gamma_{i}(T)=\Gamma_{i 0}+C\left(1+\frac{2}{e^{\hbar \omega_{i 0} / 2 k_{B} T}-1}\right),
\end{aligned}
$$

where $\omega_{i 0}$ and $\Gamma_{i 0}$ are quasiharmonic frequency and full width (instrument limited), respectively. $k_{B}$ is the Boltzmann constant, $T$ represents the temperature, and $A$ and $C$ are anharmonic constants.

Part of the phonons [Figs. 5(a)-5(d)] reveals anomalous frequency shifts while FWHMs were well described by anharmonic curves thus obeying the characteristic behavior in the case of spin-phonon interaction [57]. It is also curious to note that all the modes subject to such interaction undergo softening (negative spin-phonon coupling constants). Another 
part of phonons [Figs. 5(c) and 5(d)] manifests strong shifts by $2 \mathrm{~cm}^{-1}$ which is almost twice as large in comparison to hardening in the 10-300 K range due to the anharmonicity. Such strong spin-phonon coupling is not typical for small-spin ( $S=$ 1) systems, e.g., $\mathrm{NiWO}_{4}$ [58] and $\mathrm{Ni}_{2} \mathrm{NbBO}_{6}$ [59], however comparable and even larger shifts were observed in $\mathrm{Ni}_{3} \mathrm{TeO}_{6}$ [47] due to strong modulation of superexchange interaction by a $666 \mathrm{~cm}^{-1}$ mode which manifests as compression of the $\left[\mathrm{NiO}_{6}\right]$ octahedra in the $a b$ planes.

Experimental values of spin-phonon constants $\lambda$ are determined as a frequency difference of the phonon mode at the lowest temperature $(10 \mathrm{~K})$ and that predicted by an anharmonic equation [Eq. (3)]; they are listed in Table I. In the case of the $S=1$ ions this difference equals the spin-phonon constant $\lambda$. The contribution of spin-phonon interaction to phonon frequencies can be described by a static averaged spin-spin correlation function $\lambda\left\langle\mathbf{S}_{i} \cdot \mathbf{S}_{j}\right\rangle$ for adjacent spins [60]. This contribution within the mean field approach is expressed as $\left(\left\langle S^{z}\right\rangle / S\right)^{2}$, where $\left\langle S^{z}\right\rangle$ is the Brillouin function. Fitting of the experimental data for $\mathrm{Ni}_{2} \mathrm{InSbO}_{6}$ gives satisfactory results with spin-phonon coupling constants $\lambda$ different (or vanishing) for different phonons as shown in Fig. 5. However, obvious discrepancies of spin-phonon contributions with experimental values in the vicinity and above the Néel temperature are seen which points to important contribution of the short-range magnetic ordering. The existence of such short-range correlations in the paramagnetic phase is independently confirmed by the presence of the two-magnon mode well above $T_{N}$ [see Fig. 5(f)].

\section{E. Magnetic dynamics}

At low temperatures a pronounced broad band with maximum centered at $247 \mathrm{~cm}^{-1}$ was observed in the scattering spectra, see $Y(Z X) \bar{Y}$ spectrum in Fig. 6. This excitation was

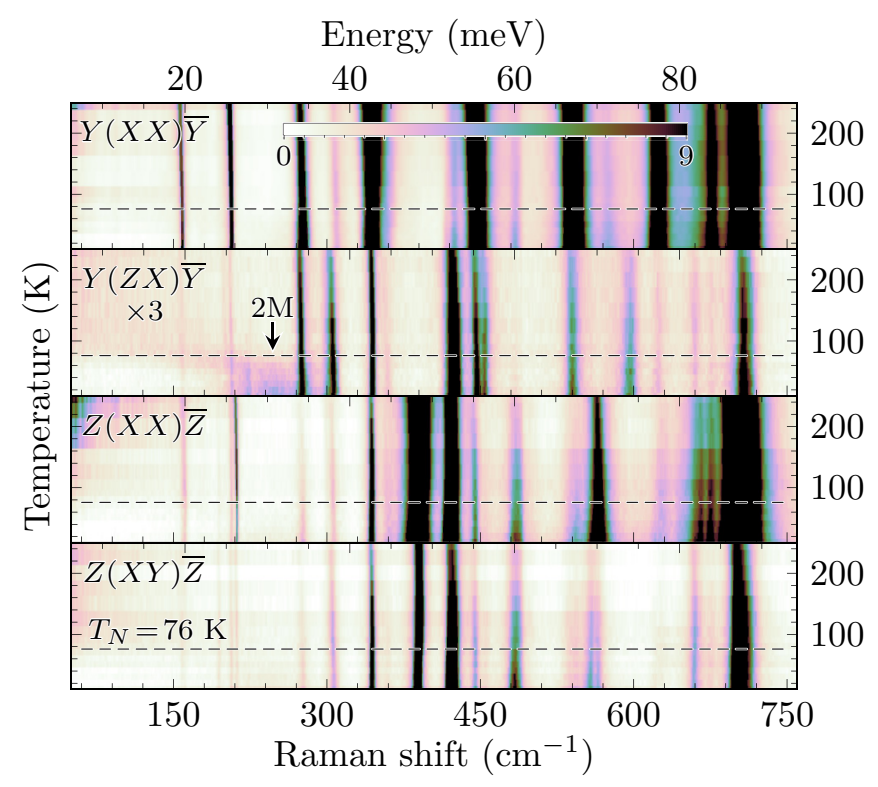

FIG. 6. Temperature maps of Raman spectra. Dashed horizontal line indicates the temperature of the antiferromagnetic phase transition at $T_{N}=76 \mathrm{~K}$. Note different scale for $Y(Z X) \bar{Y}$ map. observed in $Y(Z X) \bar{Y}, Z(X Y) \bar{Z}$, and $Z(X X) \bar{Z}$ polarizations with an absolute intensities ratio of 1.3:1.2:0.5. The band is strongly damped and the frequency softens while approaching the Néel temperature, as shown in Fig. 5(f). Nevertheless, it can be observed even above $T_{N}$ as a broad quasielastic wing up to $150 \mathrm{~K}$. Such features of this band such as temperature changes, overall half-width, and energy range suggest its twomagnon origin which is a process of simultaneous excitation of a magnon pair with the wave vectors $\boldsymbol{k}$ and $-\boldsymbol{k}$ [61]. No any other excitations of magnetic nature were observed in studied spectra.

\section{F. Linear spin-wave theory calculations}

It is known that the magnetic structure of $\mathrm{Ni}_{2} \mathrm{InSbO}_{6}$ is antiferromagnetic and incommensurate [21], however the magnetic propagation vector $\boldsymbol{k}=(0,0.036(1), 0)$ is rather small [21]. Thus, the smallness of this value allows us to do calculations accepting the following assumptions.

(i) The AFM structure is supposed to be commensurate with $\boldsymbol{k}=0$. (ii) We will assume that $J 1=J 2$ and $J 3=J 4$ which is strictly not the case, because the bond lengths for $J 1$ and $J 2$ are different, 3.76343 and $3.86013 \AA$, respectively, according to structural measurements [21]. For $J 3$ and $J 4$ exchange couplings bond lengths are equal, however they might be different because they are not symmetry related.

For testing the hypothesis of the two-magnon nature of the observed $247 \mathrm{~cm}^{-1}$ mode, the dispersion curves were numerically calculated within the linear spin-wave theory approach [62] and, subsequently they were used for the density of states (DOS) calculations. Note that dispersion curves and points in Fig. 7 are shown for the primitive (rhombohedral) basis. Calculations predict one doubly degenerate acoustic magnon mode, as expected, since there are two magnetic ions within the primitive cell.

As the first approach, only $J 1=J 2=2.7 \mathrm{meV}$ exchange constants were taken into account which resulted in a threedimensional magnetic structure with the maximum of DOS compatible with the position of the observed two-magnon band. Since there are no optical magnons to constrain this estimate of exchange constants, we may use paramagnetic Curie-Weiss temperature $\Theta=-184 \mathrm{~K}$, measured in [21]. This value represents averaged exchange couplings and for $\mathrm{Ni}_{2} \mathrm{InSbO}_{6}$ it is expressed as

$$
\Theta=-\frac{S(S+1)}{3 k_{B}} \sum_{i} z_{i} J_{i},
$$

where $S$ is the spin number, $z$ is the number of neighboring ions, and summation goes up for all exchange couplings. For the case of $J 1=J 2=2.7 \mathrm{meV}$ it gives us the value $\Theta=$ $-125 \mathrm{~K}$ which is significantly different from experimental value $\Theta=-184 \mathrm{~K}$. This discrepancy suggests that the nextneighbor exchange couplings have to be taken into account. It should be additionally noted that this approximation gives completely nonfrustrated magnetic structure which contradicts experimental observation since the frustration index is $|\Theta / N|=2.4$ [21]. This value is not big enough for considering $\mathrm{Ni}_{2} \mathrm{InSbO}_{6}$ as a frustrated magnet, but suggests the presence of non-negligible frustrations. 

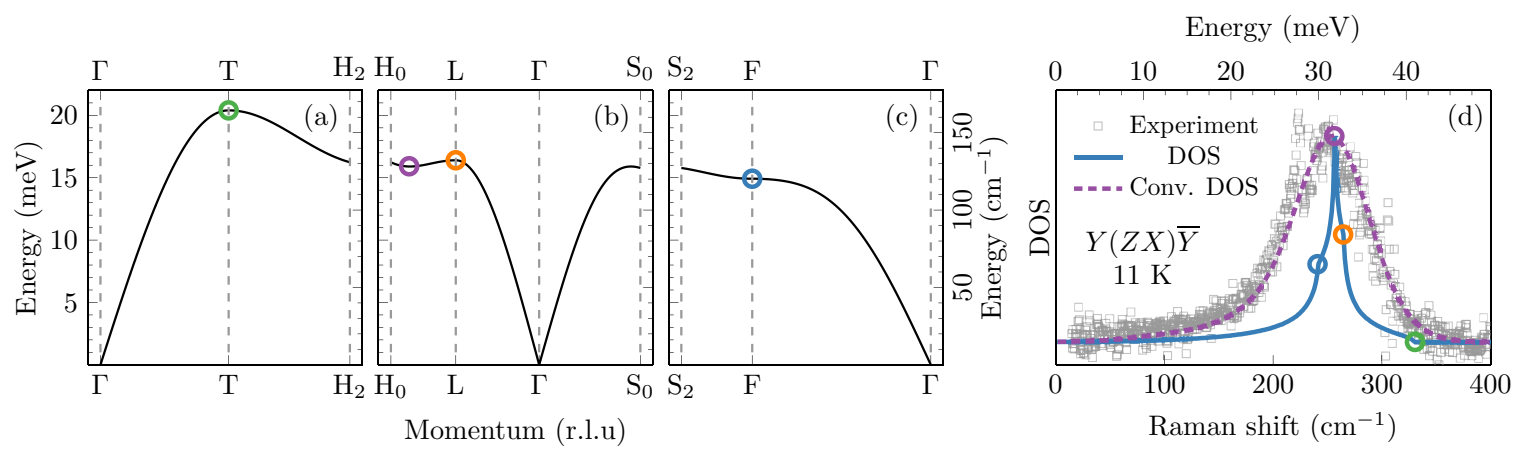

FIG. 7. (a)-(c) Spin-wave dispersion for the high-symmetry directions in the rhombohedral setting. (d) Two-magnon Raman spectrum at $T=11 \mathrm{~K}$ (gray squares) shown with the removed phonon background contribution. Magnon DOS (solid blue curve) and DOS convoluted with the Gaussian (dashed purple curve), see text for details. Note that the photon energy of DOS curves is doubled in order to match the two-magnon process. Circles highlight Van Hove singularities corresponding to particular points in the dispersion curves.

Now we include additional next-neighbor exchange paths $J 3=J 4$ connecting ions within the $X Y$ planes with Ni-Ni distances of $5.2164 \AA$ A. Though these paths connect ferromagnetically aligned spins, they were set to be antifferomagnetic in order to introduce small frustration and further decrease of $\Theta$. Such a model results in $J 1=J 2=3.4$ and $J 3=J 4=0.5 \mathrm{meV}$ and gives $\Theta=-181 \mathrm{~K}$ which is in very good agreement with the experimental value.

The Brillouin zone paths for the dispersion curves shown in Figs. 7(a)-7(c) were calculated with the use of SEEK-PATH code [63]. Since the frequency of the acoustic magnon branch is below the range achievable in our experiments, we will base our calculations on the observed two-magnon band whose position and shape should be proportional to the density of magnon states. However, it is known that in the process of the second order scattering the magnon-magnon interaction is important [64] which results in strong damping making singular points in the density of states indistinguishable. To simulate damping effects, the density of states was convoluted with Gaussian profile of $4 \mathrm{meV}$ full width. Good agreement of the calculated density of states and experiment is shown in Fig. 7(d).

No other signs of magnetic scattering were observed down to $15 \mathrm{~cm}^{-1} \approx 2 \mathrm{meV}$ which allows us to suppose that the acoustic magnon branch has energy below this value and, consequently, the single ion anisotropy is small. In fact, for the proposed exchange model the estimated value of anisotropy energy results in a value $-0.05 \mathrm{meV}$ for the case when the easy axis is directed along the $Z$ axis. This value of the anisotropy results in a gap of $\approx 2 \mathrm{meV}$ in the spin-wave spectrum at the Gamma point. Further progress in the comprehensive understanding of magnetic dynamics of $\mathrm{Ni}_{2} \mathrm{InSbO}_{6}$ crystals can be made in the studies of the antiferromagnetic resonance with the use of microwave technique or direct measurement of spin-wave dispersion by $\boldsymbol{k}$-resolved techniques, such as inelastic neutron or x-ray scattering.

\section{SUMMARY AND CONCLUSIONS}

In summary, we report the comprehensive study of a polar chiral incommensurate antiferromagnet $\mathrm{Ni}_{2} \mathrm{InSbO}_{6}$ with the use of absorption spectroscopy, optical microscopy, and polarized Raman spectroscopy. This system crystallizes in a noncentrosymmetric space group $R 3$ and undergoes a phase transition at $T_{N}=76 \mathrm{~K}$ from the paramagnetic into an incommensurate antiferromagnetic phase. Room temperature optical absorption spectroscopy revealed four bands with photon energies $1.03,1.49,1.78$, and $2.67 \mathrm{eV}$ which we assigned to ${ }^{3} A_{2} \rightarrow{ }^{3} T_{2},{ }^{1} E,{ }^{3} T_{1},{ }^{1} T_{2}$ electronic transitions within the $3 d^{8}$ states of $\mathrm{Ni}^{2+}$ ions split by the crystal field. Optical microscopy allowed us to measure the rotation of plane polarized light traversing a single crystal sample due to the natural optical activity. Two types of chiral domains related to each other by the inversion were distinguished due to the polarization rotation of opposite sign. Most phonons predicted by the group-theoretical analysis, including the split LO-TO phonons, were observed. Raman tensor elements and phase differences were determined for the $A$-symmetry modes by performing angular-resolved azimuthal polarization measurements. Temperature measurements revealed pronounced spin-phonon interaction manifested as softening of particular modes below and slightly above $T_{N}$ due to short-range magnetic ordering. A wide asymmetric two-magnon band with a maximum of $247 \mathrm{~cm}^{-1}(T=11 \mathrm{~K})$ was observed in Raman spectra with $Y(Z X) \bar{Y}, Z(X Y) \bar{Z}$, and $Z(X X) \bar{Z}$ polarizations. Based on the observed two-magnon excitation and the known value of paramagnetic Curie-Weiss temperature we propose an exchange model of the magnetic structure with parameters $J 1=J 2=3.4$ and $J 3=J 4=0.5 \mathrm{meV}$ which were used for calculating dispersion curves and density of states within linear spin theory. Our results contribute to a better understanding of lattice and magnetic dynamics in polar magnets with corundum-type structures and the developed model of exchange structure and spin-wave dispersion curves can be directly applied to other similar systems.

\section{ACKNOWLEDGMENT}

This work was funded by the Russian Science Foundation according to the research Project No. 16-12-10456. 
[1] I. Sirotin and M. Shaskolskaya, Fundamentals of Crystal Physics (MIR, Moscow, 1982).

[2] J. F. Nye, Physical Properties of Crystals: Their Representation by Tensors and Matrices (Oxford University Press, Oxford, 1985).

[3] Y.-R. Shen, The Principles of Nonlinear Optics (WileyInterscience, New York, 1984).

[4] W. Zhong, R. D. King-Smith, and D. Vanderbilt, Giant LO-TO Splittings in Perovskite Ferroelectrics, Phys. Rev. Lett. 72, 3618 (1994).

[5] J. Hlinka, J. Pokorny, S. Karimi, and I. M. Reaney, Angular dispersion of oblique phonon modes in $\mathrm{BiFeO}_{3}$ from micro-Raman scattering, Phys. Rev. B 83, 020101(R) (2011).

[6] M. Saito, K. Taniguchi, and T.-h. Arima, Gigantic optical magnetoelectric effect in $\mathrm{CuB}_{2} \mathrm{O}_{4}$, J. Phys. Soc. Jpn. 77, 013705 (2008).

[7] Y. Nii, R. Sasaki, Y. Iguchi, and Y. Onose, Microwave magnetochiral effect in the non-centrosymmetric magnet $\mathrm{CuB}_{2} \mathrm{O}_{4}$, J. Phys. Soc. Jpn. 86, 024707 (2017).

[8] T. Nomura, X.-X. Zhang, S. Zherlitsyn, J. Wosnitza, Y. Tokura, N. Nagaosa, and S. Seki, Phonon Magnetochiral Effect, Phys. Rev. Lett. 122, 145901 (2019).

[9] E. V. Gomonay and V. M. Loktev, Spintronics of antiferromagnetic systems, Low Temp. Phys. 40, 17 (2014).

[10] T. Jungwirth, X. Marti, P. Wadley, and J. Wunderlich, Antiferromagnetic spintronics, Nat. Nanotechnol. 11, 231 (2016).

[11] V. Baltz, A. Manchon, M. Tsoi, T. Moriyama, T. Ono, and Y. Tserkovnyak, Antiferromagnetic spintronics, Rev. Mod. Phys. 90, 015005 (2018).

[12] P. Němec, M. Fiebig, T. Kampfrath, and A. V. Kimel, Antiferromagnetic opto-spintronics, Nat. Phys. 14, 229 (2018).

[13] H. Watanabe, T. Kurihara, T. Kato, K. Yamaguchi, and T. Suemoto, Observation of long-lived coherent spin precession in orthoferrite $\mathrm{ErFeO}_{3}$ induced by terahertz magnetic fields, Appl. Phys. Lett. 111, 092401 (2017).

[14] R. V. Pisarev, Crystal optics of magnetoelectrics, Ferroelectrics 162, 191 (1994).

[15] R. V. Pisarev, Broken symmetries and optical phenomena in crystals, Ferroelectrics 183, 39 (1996).

[16] T.-H. Arima, Magneto-electric optics in non-centrosymmetric ferromagnets, J. Phys.: Condens. Matter 20, 434211 (2008).

[17] S. Dong, J.-M. Liu, S.-W. Cheong, and Z. Ren, Multiferroic materials and magnetoelectric physics: Symmetry, entanglement, excitation, and topology, Adv. Phys. 64, 519 (2015).

[18] S.-W. Cheong, D. Talbayev, V. Kiryukhin, and A. Saxena, Broken symmetries, non-reciprocity, and multiferroicity, npj Quantum Mater. 3, 19 (2018).

[19] Y. Tokura and N. Nagaosa, Nonreciprocal responses from noncentrosymmetric quantum materials, Nat. Commun. 9, 3740 (2018).

[20] T. J. Sato and K. Matan, Nonreciprocal Magnons in Noncentrosymmetric Magnets, J. Phys. Soc. Jpn. 88, 081007 (2019).

[21] S. A. Ivanov, R. Mathieu, P. Nordblad, R. Tellgren, C. Ritter, E. Politova, G. Kaleva, A. Mosunov, S. Stefanovich, and M. Weil, Spin and dipole ordering in $\mathrm{Ni}_{2} \mathrm{InSbO}_{6}$ and $\mathrm{Ni}_{2} \mathrm{ScSbO}_{6}$ with corundum-related structure, Chem. Mater. 25, 935 (2013).

[22] G.-H. Cai, M. Greenblatt, and M.-R. Li, Polar magnets in double corundum oxides, Chem. Mater. 29, 5447 (2017).
[23] S. Vasala and M. Karppinen, $A_{2} B^{\prime} B^{\prime \prime} O_{6}$ perovskites: A review, Prog. Solid State Chem. 43, 1 (2015).

[24] R. E. Newnham and E. P. Meagher, Crystal structure of $\mathrm{Ni}_{3} \mathrm{TeO}_{6}$, Mater. Res. Bull. 2, 549 (1967).

[25] R. Becker and H. Berger, Reinvestigation of $\mathrm{Ni}_{3} \mathrm{TeO}_{6}$, Acta Crystallogr. Sec. E: Structure Rep. Online 62, i222 (2006).

[26] J. W. Kim, S. Artyukhin, E. D. Mun, M. Jaime, N. Harrison, A. Hansen, J. J. Yang, Y. S. Oh, D. Vanderbilt, V. S. Zapf, and S.-W. Cheong, Successive Magnetic-Field-Induced Transitions and Colossal Magnetoelectric Effect in $\mathrm{Ni}_{3} \mathrm{TeO}_{6}$, Phys. Rev. Lett. 115, 137201 (2015).

[27] I. Živković, K. Prša, O. Zaharko, and H. Berger, $\mathrm{Ni}_{3} \mathrm{TeO}_{6}-$ A collinear antiferromagnet with ferromagnetic honeycomb planes, J. Phys.: Condens. Matter 22, 056002 (2010).

[28] C. Kranert, C. Sturm, R. Schmidt-Grund, and M. Grundmann, Raman Tensor Formalism for Optically Anisotropic Crystals, Phys. Rev. Lett. 116, 127401 (2016).

[29] M. Grundmann, C. Sturm, C. Kranert, S. Richter, R. SchmidtGrund, C. Deparis, and J. Zúñiga-Pérez, Optically anisotropic media: New approaches to the dielectric function, singular axes, microcavity modes and Raman scattering intensities, Phys. Status Solidi (RRL) Rapid Res. Lett. 11, 1600295 (2017).

[30] W. Zheng, J. Yan, F. Li, and F. Huang, Elucidation of "phase difference" in Raman tensor formalism, Photon. Res. 6, 709 (2018).

[31] J. Kim, J.-U. Lee, J. Lee, H. J. Park, Z. Lee, C. Lee, and H. Cheong, Anomalous polarization dependence of Raman scattering and crystallographic orientation of black phosphorus, Nanoscale 7, 18708 (2015).

[32] M. Li, Y. Wu, T. Li, Y. Chen, H. Ding, Y. Lin, N. Pan, and X. Wang, Revealing anisotropy and thickness dependence of Raman spectra for SnS flakes, RSC Adv. 7, 48759 (2017).

[33] T. Strach, J. Brunen, B. Lederle, J. Zegenhagen, and M. Cardona, Determination of the phase difference between the Raman tensor elements of the $A_{1 g}$-like phonons in $\mathrm{SmBa}_{2} \mathrm{Cu}_{3} \mathrm{O}_{7-\delta}$, Phys. Rev. B 57, 1292 (1998).

[34] C. Kranert, C. Sturm, R. Schmidt-Grund, and M. Grundmann, Raman tensor elements of $\beta-\mathrm{Ga}_{2} \mathrm{O}_{3}$, Sci. Rep. 6, 35964 (2016).

[35] A. Togo and I. Tanaka, First principles phonon calculations in materials science, Scr. Mater. 108, 1 (2015).

[36] M. Weil, R. Mathieu, P. Nordblad, and S. A. Ivanov, Crystal growth experiments in the systems $\mathrm{Ni}_{2} \mathrm{MSbO}_{6}(\mathrm{M}=\mathrm{Sc}$, In) using chemical vapour transport reactions: $\mathrm{Ni}_{2} \mathrm{InSbO}_{6}$ and $\mathrm{NiSb}_{2} \mathrm{O}_{6}$ crystals in the millimetre range, Cryst. Res. Technol. 49, 142 (2014).

[37] A. B. P. Lever, Inorganic Electronic Spectroscopy, 2nd ed. (Elsevier, Amsterdam, 1984).

[38] J. Hugel and M. Belkhir, Nature of the NiO absorption edge within a spin polarized band scheme, Solid State Commun. 73, 159 (1990).

[39] R. G. Burns, Mineralogical Applications of Crystal Field Theory (Cambridge University Press, Cambridge, UK, 1993), Vol. 5.

[40] R. V. Pisarev, M. A. Prosnikov, V. Yu. Davydov, A. N. Smirnov, E. M. Roginskii, K. N. Boldyrev, A. D. Molchanova, M. N. Popova, M. B. Smirnov, and V. Y. Kazimirov, Lattice dynamics and a magnetic-structural phase transition in the nickel orthoborate $\mathrm{Ni}_{3}\left(\mathrm{BO}_{3}\right)_{2}$, Phys. Rev. B 93, 134306 (2016). 
[41] A. D. Molchanova, Experimental study and analysis of absorption spectra of $\mathrm{Ni}^{2+}$ ions in nickel orthoborate $\mathrm{Ni}_{3}\left(\mathrm{BO}_{3}\right)_{2}$, Phys. Solid State 60, 1957 (2018).

[42] R. Newman and R. M. Chrenko, Optical properties of nickel oxide, Phys. Rev. 114, 1507 (1959).

[43] M. Fiebig, D. Fröhlich, T. Lottermoser, V. V. Pavlov, R. V. Pisarev, and H.-J. Weber, Second Harmonic Generation in the Centrosymmetric Antiferromagnet NiO, Phys. Rev. Lett. 87, 137202 (2001).

[44] M. O. Yokosuk, A. al-Wahish, S. Artyukhin, K. R. ONeal, D. Mazumdar, P. Chen, J. Yang, Y. S. Oh, S. A. McGill, K. Haule, S. W. Cheong, D. Vanderbilt, and J. L. Musfeldt, Magnetoelectric Coupling through the Spin Flop Transition in $\mathrm{Ni}_{3} \mathrm{TeO}_{6}$, Phys. Rev. Lett. 117, 147402 (2016).

[45] M. I. Aroyo, J. M. Perez-Mato, D. Orobengoa, E. Tasci, G. De La Flor, and A. Kirov, Crystallography online: Bilbao crystallographic server, Bulg. Chem. Commun 43, 183 (2011).

[46] X. Wang, F.-T. Huang, J. Yang, Y. S. Oh, and S.-W. Cheong, Interlocked chiral/polar domain walls and large optical rotation in $\mathrm{Ni}_{3} \mathrm{TeO}_{6}$, APL Mater. 3, 076105 (2015).

[47] M. O. Yokosuk, S. Artyukhin, A. al-Wahish, X. Wang, J. Yang, Z. Li, S.-W. Cheong, D. Vanderbilt, and J. L. Musfeldt, Tracking the continuous spin-flop transition in $\mathrm{Ni}_{3} \mathrm{TeO}_{6}$ by infrared spectroscopy, Phys. Rev. B 92, 144305 (2015).

[48] G. Blasse and W. Hordijk, The vibrational spectrum of $\mathrm{Ni}_{3} \mathrm{TeO}_{6}$ and $\mathrm{Mg}_{3} \mathrm{TeO}_{6}$, J. Solid State Chem. 5, 395 (1972).

[49] W. Hayes and R. Loudon, Scattering of Light by Crystals (Courier Corporation, North Chelmsford, MA, 2012).

[50] To include trigonal symmetry of the system, $\Theta \rightarrow \frac{2}{3} \Theta$ substitution was made for $Z(X X) \bar{Z} \leftrightarrow Z(Y Y) \bar{Z}$ scattering geometries.

[51] S. Margueron, A. Bartasyte, A. M. Glazer, E. Simon, J. Hlinka, I. Gregora, and J. Gleize, Resolved e-symmetry zone-centre phonons in $\mathrm{LiTaO}_{3}$ and $\mathrm{LiNbO}_{3}$, J. Appl. Phys. 111, 104105 (2012).

[52] R. H. Lyddane, R. G. Sachs, and E. Teller, On the polar vibrations of alkali halides, Phys. Rev. 59, 673 (1941).

[53] These ratios are 1.02, 1.03, 1.14, 1.04, 1.07, 1.03, and 1.01 for all the LO-TO pairs from Table I.
[54] W. Otaguro, E. Wiener-Avnear, C. A. Arguello, and S. P. S. Porto, Phonons, polaritons, and oblique phonons in $\mathrm{LiIO}_{3}$ by Raman scattering and infrared reflection, Phys. Rev. B 4, 4542 (1971).

[55] C. M. Hartwig, E. Wiener-Avnear, J. Smit, and S. P. S. Porto, Phonon Coupling and Angular Dispersion in Biaxial $\mathrm{NaNO}_{2}$, Phys. Rev. B 3, 2078 (1971).

[56] M. Balkanski, R. F. Wallis, and E. Haro, Anharmonic effects in light scattering due to optical phonons in silicon, Phys. Rev. B 28, 1928 (1983).

[57] The anharmonic constants obtained through a fitting procedure are: $\omega_{0}=346.31, A=-1.41, \Gamma_{0}=0.77, C=2.08$; $\omega_{0}=487.11, A=-2.05, \Gamma_{0}=5.15, C=7.01 ; \omega_{0}=392.31$, $A=-1.06, \Gamma_{0}=1.08, C=2.02 ; \quad \omega_{0}=420.01, A=-0.57$, $\Gamma_{0}=1.88, C=1.37 ; \quad \omega_{0}=207.70, A=-0.82, \Gamma_{0}=1.28$, $C=1.26$ for curves (a)-(e) in Fig. 5, respectively.

[58] M. A. Prosnikov, V. Y. Davydov, A. N. Smirnov, M. P. Volkov, R. V. Pisarev, P. Becker, and L. Bohatý, Lattice and spin dynamics in a low-symmetry antiferromagnet $\mathrm{NiWO}_{4}$, Phys. Rev. B 96, 014428 (2017).

[59] M. A. Prosnikov, A. N. Smirnov, V. Yu. Davydov, R. V. Pisarev, N. A. Lyubochko, and S. N. Barilo, Magnetic dynamics and spin-phonon coupling in the antiferromagnet $\mathrm{Ni}_{2} \mathrm{NbBO}_{6}$, Phys. Rev. B 98, 104404 (2018).

[60] D. J. Lockwood and M. G. Cottam, The spin-phonon interaction in $\mathrm{FeF}_{2}$ and $\mathrm{MnF}_{2}$ studied by Raman spectroscopy, J. Appl. Phys. 64, 5876 (1988).

[61] P. A. Fleury and R. Loudon, Scattering of light by one-and twomagnon excitations, Phys. Rev. 166, 514 (1968).

[62] S. Toth and B. Lake, Linear spin wave theory for single-Q incommensurate magnetic structures, J. Phys.: Condens. Matter 27, 166002 (2015).

[63] Y. Hinuma, G. Pizzi, Y. Kumagai, F. Oba, and I. Tanaka, Band structure diagram paths based on crystallography, Comput. Mater. Sci. 128, 140 (2017).

[64] R. J. Elliott and M. F. Thorpe, The effects of magnon-magnon interaction on the two-magnon spectra of antiferromagnets, J. Phys. C 2, 1630 (1969). 